\title{
Development of a Wearable Haptic Display for Situation Awareness in Altered-gravity Environment: Some Initial Findings
}

\author{
Ryan Traylor and Hong Z. Tan \\ Haptic Interface Research Laboratory \\ Purdue University \\ 1285 EE Building, West Lafayette, IN 47907-1285 \\ \{traylorr,hongtan\}@purdue.edu
}

\begin{abstract}
Efforts are under way to develop a wearable haptic display that can impart directional information on a user's back for situation awareness. To date, two studies have been conducted aboard the NASA KC-135A reduced gravity aircraft to investigate the perception of tactile information in altered-gravity environments. This paper reports our results on perceived loudness of vibrotactile stimulation under different gravity conditions. Subjects compared seven fixed-frequency varying-amplitude vibrations in 1.8-g to a reference vibration delivered in zero-g using the method of constant stimuli. Our results show that the points of subjective equality (PSE) measured in 1.8-g are essentially the same as the intensity of the reference signal delivered in zero-g. The difference between PSE and the reference is less than the difference threshold $(D L)$ measured in 1.8-g. We also found that the displacements (measured with an accelerometer) produced by our tactors in one-g and zero-g conditions are the same using identical driving waveforms. Our data suggest that the perceived loudness of vibrotactile stimuli remains the same in altered-gravity environments. However, a user's ability to interpret vibrotactile signals in zero-g environment may be hampered by increased cognitive load due to the need to continuously monitor the position and movement of one's body.
\end{abstract}

\section{Introduction}

For the past several years, we have been developing a wearable haptic display that can be sewn into the back of a vest. We envision such a system to be useful for navigation guidance and situation awareness (Ertan, Lee, Willets, Tan, \& Pentland, 1998) (Tan \& Pentland, 2001). A haptic display can be invaluable when a user's visual/auditory system is overloaded or when visual/auditory information is obscure (e.g., pilots, divers, blind travelers). One potential application that we have focused on is the problem of spatial disorientation for pilots. Spatial disorientation refers to the incorrect perception of attitude, altitude, or motion of one's own aircraft relative to the earth or other significant objects. It typically occurs when the pilot's visual system is compromised by temporary distractions, increased workload, reduced visibility, and g-lock (a pilot undergoes a high-g maneuver and temporarily blacks out behind the stick). Many systems have been proposed to reduce the incidents of spatial disorientation mishaps. Among those, the TSAS (tactile situation awareness system, developed by Rupert and colleagues) is perhaps the most fully implemented and tested system (Rupert, 2000). Navy test pilots have successfully performed a variety of maneuvers (including acrobatics) using the TSAS as the sole source of information regarding the state of the aircraft.

While the TSAS display uses about one hundred pneumatic tactors that are densely spaced over a pilot's body suite, our wearable haptic display contains a coarse 3-by-3 array of vibrotactile stimulators with $10 \mathrm{~cm}$ interelement spacing. While the TSAS uses the location of individual tactors to indicate information (such as pitch and roll) to its user, our haptic display imparts directional information based on the "sensory saltation" phenomenon. Sensory saltation is a perceptual illusion that, when evoked, creates a vivid and compelling sense of direction (Geldard, 1975). Our previous study has shown that untrained observers can correctly identify one of eight directions (east, west, north, south, northeast, northwest, southeast, and southwest) with an accuracy of 79 to $91 \%$ (Tan, Lim, \& Traylor, 2000).

In August 1999, a group of four Purdue undergraduate students constructed a wearable vest (with embedded tactor array and the associated electronics) and tested its utility aboard a NASA KC-135A reduced-gravity aircraft. The core of the apparatus was a 3-by-3 tactor array attached to the back of the vest (see inset in Fig. 1). The students were trained with four directional signals (east, west, north, and south) and achieved close to perfect 
identification performance prior to the flights. During the zero-gravity portions of the KC-135A flights, the students were presented with one of the four directional signals on their back, and were asked to indicate the perceived direction relative to the torso. Our goal was to test whether saltatory signals could be well perceived in a zero-gravity environment. The results from two subjects showed that the overall identification accuracy was $44 \%$ in zero-g, as compared to close to $100 \%$ in one-g from the same subjects. The students reported that the vibratory signals felt considerably weaker in zero-g than in one-g.

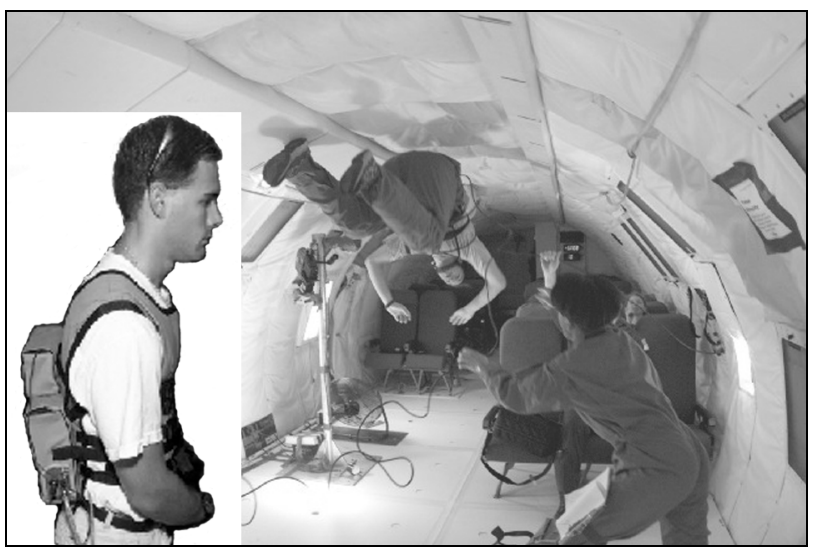

Figure 1. Purdue students Adrian Lim (front right) and Joachim Deguara (flying) conducted direction

identification experiments in zero-g environment. The inset shows Ryan Casteel wearing the vest with

embedded tactor array, and the backpack containing all the electronics and battery packs.

Post-flight analysis converged on three main factors that might have contributed to the relatively low direction identification accuracy. First, the hardware might have functioned differently in zero-g. Although we had no reason to suspect the tactors to work differently in zero-g, we nevertheless did not have any measurement data to prove so. Second, perceived loudness of vibrotactile stimulation might have changed in zero-g due to factors such as body fluid shift. Although there have been studies investigating the use of haptic interfaces in microgravity (Payette, Hayward, Ramstein, \& Bergeron, 1996), there is, to the best of our knowledge, no published literature on whether tactile thresholds change in zero-g. Third, the subjects might have been distracted and therefore unable to devote their full attention to the vibrotactile stimuli. Flying in zero-g was a new and exciting experience for the students involved. It turned out to be challenging to perform the experiment while trying to achieve a stable position with one's body in zero-g (see Fig. 1).

The results reported in this paper are from a follow up study that took place aboard the NASA KC-135A in March 2001. This study was designed specifically to address the first two factors discussed in the previous paragraph. To measure the performance of the hardware, accelerometer data from the tactor were taken during the zero-g portion of the flight. To assess the potential change in perceptual thresholds, subjects compared the perceived loudness of vibrations in zero-g and $1.8-\mathrm{g}$ environments. We were unable to directly test the third factor (i.e., cognitive load) as none of the student participants had gone through enough $\mathrm{KC}-135 \mathrm{~A}$ flights to be considered "experienced" in zero-g environment.

\section{Methods}

\subsection{Apparatus}

The KC-135A simulates altered gravity environments by flying through a series of maneuvers called parabolas. The plane begins each parabola at around $24,000 \mathrm{ft}$. by climbing rapidly at a 45 degree angle. As the plane reaches an altitude of around 32,000 ft., it begins to freefall and follows the peak of the parabola, which creates a microgravity environment inside the aircraft. Next, the plane comes out of freefall and descends at a 45 degree angle. Finally the plane flies through the trough of the parabola thereby creating a $1.8-\mathrm{g}$ environment. Each zero-g and 1.8-g portion of the parabola has a duration of approximately 25 seconds. During a typical flight such as ours, the aircraft flies 30 parabolas. More details can be found at http://zerog.jsc.nasa.gov/home.html.

The hardware used to drive the tactor and to measure signal intensity consists of five main components: the tactor, accelerometer, keypad, microcontroller, and tactor driver circuit (Fig. 2). The tactile display consists of a single tactor strapped to the volar side of the user's forearm. The tactor is modified from a flat speaker, four centimeters in diameter, to bring its resonant frequency down to around $300 \mathrm{~Hz}$ (Audiological Engineering Corp., Somerville, MA). An accelerometer (ACH-01-03, by Measurement Specialties Inc., Fairfield, NJ) is placed on top of the tactor (as shown in Fig. 2) to record the displacement of the tactor during signal delivery. The keypad and its associated electronic display (LED's) is used as a simple user interface to facilitate interaction with the data collection program.

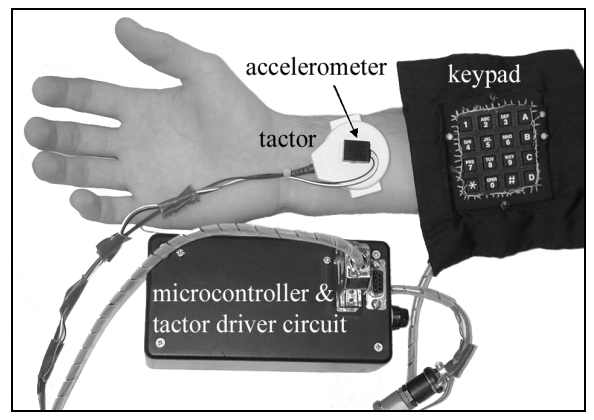

Figure 2. Illustration of main hardware components. 
The microcontroller directs information to and from the user, controls the intensity of the signal sent to the tactor, and records the signals from the accelerometer. It is programmed to selectively present one of seven vibrational signals of various intensities to the tactor when prompted by the user. The microcontroller records the user's responses in EEPROM, so that its contents are retained even if electrical power is somehow disconnected during flight. Data is also gathered from the accelerometer by the microcontroller's on-chip A/D converter and stored in SRAM.

The main function of the tactor driver circuit is to supply an amplified oscillating signal to the tactor when an enable signal is received from the microcontroller. The circuit consists of an oscillator, power supply, and bridge amplifier. The amplitude of this oscillating signal is governed by the voltage level on a control line generated by the microcontroller.

The overall hardware system is designed to be selfcontained and easily operated by one person, since the students are not allowed to put on any test equipment until they are airborne. Once in the air and right before the KC-135A starts its first parabola, the subjects put the tactor and the accelerometer under a wide elastic band worn on the forearm (see Fig. 3). The keypad is placed on the forearm near the elbow. The control box containing the microcontroller and tactor driver circuitry is strapped to the upper arm. Finally, the battery pack is placed around the waist of the subject.

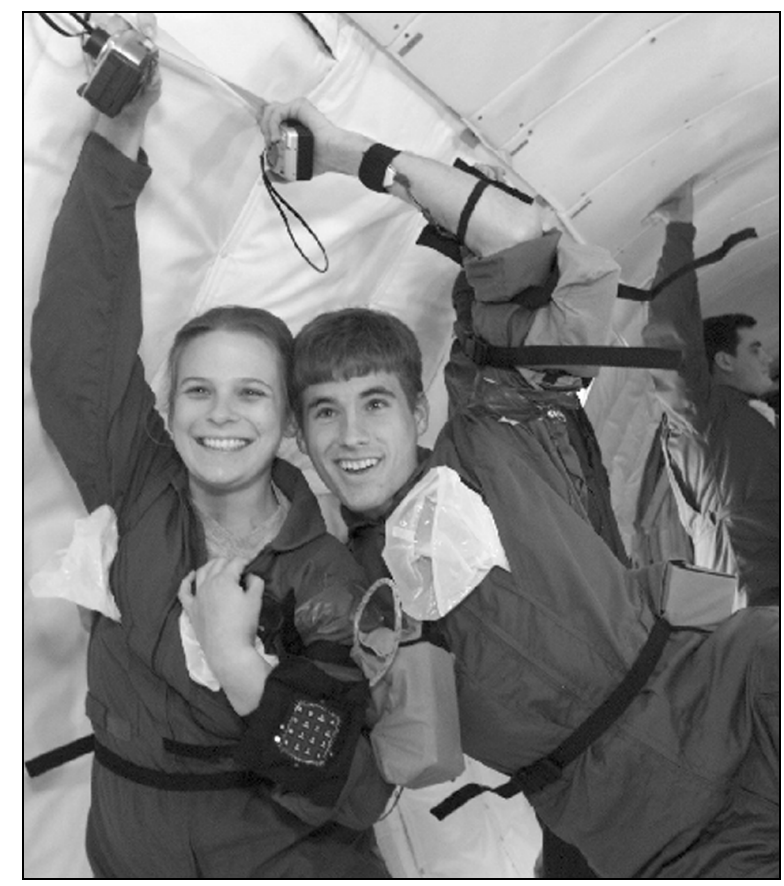

Figure 3. Lauren Naessens and Ryan Traylor equipped with the experimental apparatus aboard KC-135A in March 2001.

\subsection{Subject}

Four Purdue undergraduate students, three males (R, D, and $\mathrm{J}$ ) and one female (L), participated in this study. They are all members of our flight team with ages between 21 to 22 years old. Subjects D and J flew the first day while subjects $\mathrm{L}$ and $\mathrm{R}$ flew the following day in March 2001. Among the four subjects, only J had participated in an earlier flight in August 1999.

\subsection{Stimulus}

The stimuli used in this study are seven fixedfrequency 250-ms long sinusoidal signals that vary in their intensities. Due to the fact that NASA allows two students per team per KC-135A flight, two sets of hardware are constructed so that both subjects can perform experiments. The characteristics of the two hardware systems are slightly different due to varying electrical tolerances in the components. Therefore, each subject was assigned to a specific set of hardware and used it exclusively throughout pre-flight, in-flight and post-flight experiments. Subjects D and R used hardware set 1 , and subjects $\mathrm{J}$ and $\mathrm{L}$ used set 2 . The tactors in sets 1 and 2 are driven at their respective resonant frequencies of 310 and $279 \mathrm{~Hz}$.

The intensities of each tactor are calibrated with an accelerometer in one-g condition and expressed in $\mathrm{dB}$ SL (i.e., $\mathrm{dB}$ above the human absolute detection threshold at the corresponding frequency). Due to the fact that detection thresholds for vibrations vary with body sites and tactor contact areas, and that most published data are for contact areas much smaller than the one we used (Verrillo \& Gescheider, 1992), we measured the detection thresholds for our tactors at their respective resonant frequencies with two subjects using the method of adjustment. The resultant threshold of $-16 \mathrm{~dB}$ (re $1 \mu \mathrm{m}$ peak) was used to compute the sensation levels for all stimuli. These values are shown in Table 1. Note that the minimum and maximum values of the stimulus intensity per hardware set is limited by the combinations of resistor values that can be realized in the circuitry. Efforts were made towards placing all seven stimuli per hardware set on a more or less linear scale in units of dB SL.

Table 1. Stimulus intensities for the two hardware sets. Numbers with asterisks denote the reference signals.

\begin{tabular}{|c|c|c|c|c|c|c|c|}
\hline Set & \multicolumn{5}{|c|}{ Intensity of Seven Stimulus Alternatives (dB SL) } \\
\hline 1 & 20.29 & 23.74 & 26.10 & 27.17 & $28.76^{*}$ & 30.22 & 31.69 \\
\hline 2 & 17.72 & 22.42 & 25.35 & 27.26 & $29.90^{*}$ & 31.75 & 34.66 \\
\hline
\end{tabular}

\subsection{Procedure}

The method of constant stimuli was used for measuring the point of subjective equality (PSE) and difference threshold (DL). In a typical session, a subject felt the reference signal first. The subject was then presented with 
one of the seven stimuli and asked to respond whether the test signal felt weaker or stronger than the reference.

Prior to the $\mathrm{KC}-135 \mathrm{~A}$ flights, all four subjects were tested with reference signals set at 28.76 and $29.90 \mathrm{~dB}$ SL for hardware sets 1 and 2, respectively (see numbers with asterisks in Table 1). These preflight tests served to train our subjects with the experimental protocol and with the use of the keypad for interacting with the microcontroller.

To address the issue of whether perceived loudness of vibrotactile stimulation changes with the gravity condition, the reference and the test vibrations should be delivered under different gravity conditions. It was deemed impractical to deliver the reference signal prior to each flight and expect the subjects to memorize the reference signal level throughout the thirty parabola flights. Therefore, we decided to compare vibrations delivered between the zero-g and the 1.8-g portions of the parabolas. Based on the experience gained from our previous flights, we chose to deliver the reference signal in zero-g when the subjects were flying around in the air, and perform the method of constant stimuli experiment in 1.8-g when their bodies were "glued" to the floor of the aircraft (and hence it was easier for them to operate the keypad). With these considerations, the following procedure was repeated for each parabola aboard the $\mathrm{KC}$ $135 \mathrm{~A}$.

When the plane entered into the zero-g portion of a parabola, the subject pressed the "zero-g" key on the keypad. The $250 \mathrm{~ms}$ reference signal (see Table 1) was presented to the subject once every second. When the zero-g period was over, the subject pressed the "exit" key. As the plane entered into the 1.8-g portion of the parabola, the user pressed the "1.8-g" key on the keypad. Shortly thereafter, the tactor vibrated once for $250 \mathrm{~ms}$ with an intensity randomly chosen from the seven alternatives. The subject responded by pressing either the "stronger" or the "weaker" key on the keypad. Another randomly selected stimulus was then presented, until the subject pressed the "exit" key to indicate the end of the 1.8-g environment.

During one of the zero-g periods, the subject pressed a special key sequence to initiate data sampling from the accelerometer, using a calibration waveform as the input.

\subsection{Data Analysis}

Data from the method of constant stimuli are analyzed with the probit analysis routine in the SAS software package. The probit analysis essentially fits a cumulative Gaussian function to the proportions of trials with the "stronger" response. The mean and the standard deviation of the Gaussian are then taken as the point of subjective equality (PSE) and the difference threshold (DL), respectively. To the extent that the difference between the intensity of the reference signal (delivered during zero-g) and PSE (estimated with data collected during 1.8-g) exceeded DL, we can conclude that perceived loudness changed from zero-g to 1.8 -g environment.

The amplitude of the accelerometer data (a sinusoidal function) is converted to its equivalent value in displacement. The amplitudes of vibrations in one-g and zero-g for the same calibration input waveform are then compared.

\section{Results}

Experimental results from the preflight tests in one-g condition are listed in Table 2. As expected, the PSE values are in close alignment with the reference signal intensity. The average values for |PSE - Reference| and DL are 0.61 and $2.13 \mathrm{~dB}$ SL, respectively. Since the average value of |PSE - Reference| is well within that of DL, we conclude that there is no bias in the judgments of subjective loudness of vibrotactile intensities in one-g.

Table 2. Point of subjective equality (PSE) and difference threshold (DL) results from preflight (one-g) experiments. All entries are expressed in dB SL relative to absolute thresholds measured in one-g condition.

\begin{tabular}{|c|c|c|c|c|}
\hline Subject & Reference & No. of Trials & PSE & DL \\
\hline D & 28.76 & 598 & 27.36 & 1.66 \\
\hline J & 29.90 & 596 & 30.38 & 2.28 \\
\hline L & 29.90 & 602 & 29.63 & 2.61 \\
\hline R & 28.76 & 263 & 28.47 & 1.95 \\
\hline
\end{tabular}

Results from the KC-135A flights are summarized in Table 3. It is clear that the PSE values are very close to the reference signal values, except for subject $J$. This subject ( $\mathrm{J}$ ) had a particularly large percentage of responding "stronger" at the lowest stimulus intensity (22.7\% at $17.72 \mathrm{~dB}$ SL), which may have contributed to a less accurate fit of the cumulative Gaussian curve. The average values for |PSE - Reference| and DL are 2.62 and $4.07 \mathrm{~dB}$ SL, respectively. Again, the average value of |PSE - Reference| is well within that of DL. Recall that during parabola flights, the reference signal was presented during the zero-g portions of the flight, and the seven stimulus levels were delivered during the 1.8-g portions. Therefore, we conclude that the perceived loudness of vibrations does not change significantly from zero-g to 1.8-g.

Table 3. Point of subjective equality (PSE) and difference threshold (DL) results from experiments conducted in altered-g. All entries are expressed in dB SL relative to absolute thresholds measured in one-g.

\begin{tabular}{|c|c|c|c|c|}
\hline Subject & Reference & No. of Trials & PSE & DL \\
\hline D & 28.76 & 253 & 28.51 & 1.96 \\
\hline J & 29.90 & 365 & 23.77 & 6.73 \\
\hline L & 29.90 & 304 & 27.40 & 4.33 \\
\hline R & 28.76 & 312 & 27.15 & 3.25 \\
\hline
\end{tabular}


Our accelerometer measurements were taken in the one-g and zero-g environments with the same driving waveform. With an 8-bit A/D converter and after applying the necessary conversion factors, our measurements have a nominal resolution of about 0.04 $\mu \mathrm{m}$. We found that the intensity of vibration was $1.12 \mu \mathrm{m}$ (or equivalently, $0.98 \mathrm{~dB}$ re $1 \mu \mathrm{m}$ peak) under both the zero-g and one-g conditions. It should be pointed out that the measured vibration intensity changes under various loading conditions. We tried out best to equalize the conditions for measurements in the zero-g and one-g environments.

\section{Discussion}

This paper summarizes our initial results on the development of a wearable haptic display for situation awareness in altered-gravity environments. Our goal is to use the sensory saltation phenomenon to impart directional signals to a user's back. These directional signals can potentially provide information about spatial orientation either as a redundant source of information or as the sole display when the user's visual/auditory sensory channels are overloaded or temporarily non-functional. We have so far conducted two studies aboard the NASA KC-135A reduced gravity aircraft. On our first flight, we tested a user's ability to identify one of four (left, right, up and down) directions on the back in zero-g environment. The measured identification accuracy was lower than what we had obtained in our laboratory in one-g condition. On our second flight, we investigated whether this reduction in identification accuracy was due to a change in hardware performance or in human vibrotactile intensity perception in altered-gravity environment. Our results indicate that the tactors were producing the same amount of displacement given the same driving waveform in one-g and zero-g conditions, and our perceived loudness of vibrotactile signals does not change from a zero-g to a 1.8-g environment. We therefore conclude that the most likely reason for the inferior performance of direction identification in our first flights is cognitive load.

One of the authors can contest to how difficult it is to perform the simplest task in zero-g environment. During the first few parabolas he had ever flown, Ryan Traylor was interviewed by his peers on the ground via a radio link. Ryan remembers having to struggle to concentrate on what he wanted to say. He was overwhelmed by the fact that he could no longer take for granted that his feet were always on the ground. He had to use several sensory modalities (e.g., proprioception as well as vision) to maintain awareness of the position and orientation of his body. If it was difficult for Ryan to do something as natural as speaking in the midst of flying in zero-g, it must have been challenging for the members of the first flights to pay attention to directional signals on their back in microgravity. We do believe that as one becomes more experienced with navigating in microgravity, the effect of increased cognitive load should lessen. For example, the NASA safety officers aboard the KC-135A were able to fly around effortlessly in zero-g and attend to various situations.

We plan to continue our work by participating in the NASA reduced gravity flights aboard the KC-135A. If and when possible, we would like to investigate the effect of cognitive load on haptic perception by conducting psychophyisical experiments with subjects who have varying degrees of experience in altered-gravity environments.

\section{Acknowledgment}

The authors wish to thank Dr. Roger Cholewiak for his many insightful discussions and suggestions regarding this project. We are grateful to the School of Electrical and Computer Engineering, and the Dean's Office of Schools of Engineering at Purdue University for their financial and administrative support. This work was partially funded by a National Science Foundation Faculty Early Career Development (CAREER) Award under Grant No. 9984991-IIS. We wish to thank NASA for their Reduced Gravity Student Flight Opportunities Program for this wonderful experience.

\section{References}

Ertan, S., Lee, C., Willets, A., Tan, H. Z., \& Pentland, A. (1998). A wearable haptic navigation guidance system, Digest of the Second International Symposium on Wearable Computers, 164-165.

Geldard, F. A. (1975). Sensory Saltation: Metastability in the Perceptual World. Hillsdale, New Jersey: Lawrence Erlbaum Associates.

Payette, J., Hayward, V., Ramstein, C., \& Bergeron, D. (1996). Evaluation of a force feedback (haptic) computer pointing device in zero gravity. In K. Danai (Ed.), Proceedings of the Symposium on Haptic Interfaces for Virtual Environment and Teleoperator Systems, American Society of Mechanical Engineers Dynamic Systems and Control Division, Vol. 58, 547553.

Rupert, A. H. (2000). An instrument solution for reducing spatial disorientation mishaps - A more "natural" approach to maintaining spatial orientation. IEEE Engineering in Medicine and Biology Magazine, Vol.19, 71-80.

Tan, H., Lim, A., \& Traylor, R. (2000). A psychophysical study of sensory saltation with an open response paradigm. In S. S. Nair (Ed.), Proceedings of the Ninth (9th) International Symposium on Haptic Interfaces 
for Virtual Environment and Teleoperator Systems, American Society of Mechanical Engineers Dynamic Systems and Control Division, Vol. 69-2, 1109-1115.

Tan, H. Z., \& Pentland, A. (2001). Tactual Displays for Sensory Substitution and Wearable Computers. In W. Barfield \& T. Caudell (Eds.), Fundamentals of Wearable Computers and Augmented Reality (Chap.18), 579-598.

Verrillo, R. T., \& Gescheider, G. A. (1992). Perception via the sense of touch. In I. R. Summers (Ed.), Tactile Aids for the Hearing Impaired, 1-36. 